\title{
Analysing the Insights and Assessing the Impact of a Digital Mammography and Tomosynthesis Based 2-year Long Prospective Breast Screening Programme Organised in Western India
}

\author{
Pranav Ajmera, Pratiksha Yadav*, Udayan Dosi, Shreeya Goyal
}

\begin{abstract}
Background: While the past decades have seen a rise in the number of cases diagnosed with cancer, breast cancer in particular has become the most frequently diagnosed cancer in women over the past decade. The figures for associated mortality are on a decline in most Western and developed nations, but in contrast they continue to remain high in transitional nations like India. Materials and Methods: After receiving approval by IEC and IRB, we set-up a prospective 2-year long screening programme combined with outreach camps to ensure representation of the larger population and include urban, rural and tribal population. Strict screening criteria were enforced and trained female paramedical staff were assigned to the camp for patient counselling and breast cancer awareness. Investigation was performed at the tertiary care institute utilising both full-field digital breast mammography and tomosynthesis. Biopsy was advised for highly suspicious lesions. Results: The study encompassed $n=1017$ Indian women and revealed that $39 \%(\mathrm{n}=397)$ of them belonged to 41-50 years age group. BIRADS categorisation of the lesions revealed that while majority $(57 \% ; n=580)$ women had no detectable abnormality, nearly $22 \%(n=224)$ had lesions suspected to be benign while $10 \%(\mathrm{n}=99)$ of them had lesions with a suspicion of high index of malignancy. $43 \%(\mathrm{n}=437)$ of the populace had dense breasts (type-C). Most of the BIRADS-5 lesions (36/38) were confirmed as malignant on histopathology. Conclusion: We propose a model for screening mammography and also presents the results of this programme which we implemented to screen populace from a large and densely populated geographic region. The model was successful in being self-sustainable and received a good turnout on the back of community outreach breast awareness camps and by incentivizing the women by performing mammograms completely free of cost and also providing them reports.
\end{abstract}

Keywords: Breast cancer- screening- digital breast mammography- camps- Western India

Asian Pac J Cancer Prev, 23 (1), 327-338

\section{Introduction}

Cancer is the primary cause of death worldwide and also the major barrier to increasing life expectancy. In fact, as per the World Health Organisation (WHO), it was amongst the top two causes of death before 70 years of age in almost 112 out of 183 nations. Worldwide, 19.3 million new cases were diagnosed with cancer in 2020. Amongst the massive tumor load, female breast cancer is the most commonly diagnosed cancer as per GLOBOCAN-2020 data (Sung et al., 2021). GLOBOCAN-2020 data reveals that female breast cancer is the most prevalent cancer in women, globally accounting for nearly a 2.3 million newly diagnosed cancer cases in 2020 and causing deaths in nearly $6,85,000$ women. In fact, in 2020, there were nearly a 7.8 million women diagnosed with breast cancer over the last 5 years and hence it accounted for the most number of years lost due to disability. The disease is global and is prevalent in every country afflicting women of all age groups (WHO - News-room, factsheets, detail, breast cancer, 2021). The mortality associated with breast cancer in most developed nations has shown a decrement based on the long term success of their screening programmes allowing them time to treat at an early stage when cancer has still not progressed (Cancernet- cancer types, breast cancer, statistics, n.d.). However, in the case of transitional nations, like India and other lower middle-income countries, the ground realities are in stark contrast.

The Indian subcontinent is a large peninsula with a population of over 1,380 million that is nearly $17.7 \%$ of the entire world population, amongst whom a majority live within rural regions (Worldometers-info, world population, India population, 2021). There is a nearly $35 / 75$ split of the population living in urban and rural 
regions, amongst them the females account for $48 \%$ of the population (Worldbank- indicator, Indian population, 2021). The population is relatively young with the mean age of an Indian around 28.4 years and India continues to fall within the third stage of the Demographic cycle, so the population is projected to continually expand in the near future.

In India, breast cancer has edged ahead of cervical cancer and cancer of the oral cavity to become the most common cancer and the leading cause of cancer-related deaths; the corresponding figures for the same stand at $27.7 \%$ and $11.1 \%$ respectively. The incidence of breast cancer continues to rise in India, a rise which cannot be attributed to increased screening as population-based mammographic screening programmes are still not widespread in India, so there are other potential drivers behind the uptrend in the number of cases diagnosed (McDonald et al., 2021). This problem is compounded with the higher mortality being experienced in Indian women, compared to most of the Western countries. WHO data states that the 5-year survival rate in India is a bare $66 \%$ as compared to over $90 \%$ in most Western nations and $95 \%$ in the US (WHO- Newsroom, factsheet, details, breast cancer). While direct figures for India, like the one provided by ASCO for the US, are not available, an analysis by Mathur et al., (2020) compiling data from various population-based registries and hospital-based cancer registries from India, provides supporting evidence for this abysmally low figure.

In a study published in the Lancet, the researchers, Dhillon et al., (2018) established that there was a 39.1\% increase in the age-standardised incidence rate of breast cancer in females living in India, over a 26-year study period and that breast cancer was accounting for nearly $16.8 \%$ of all Disease Adjusted Life Years (DALYs). The rise in DALYs was particularly in the age group between 30 to 60 years, after which the graph shows a downward trend.

There are significant differences in the epidemiology of breast cancer in women in India from those of the Western population (Chopra et al., 2014). While the median age at diagnosis is nearly 61 years in the Western world, and the peak age is 60-70years. In India, the peak age group occurs decades earlier between 40-50 years of age, that is many of these women are premenopausal (Chopra et al, 2014; Agarwal et al., 2017). In fact in the Indian North-Eastern population, the shift is towards an, even more, younger age group, as shown by data compiled by Chopra et al., (2014); the peak occurs almost a decade earlier than for the rest of India. In a SEER analysis performed by Kakrala et al., (2010), they concluded that Asian Indian/ Pakistani women below 40 years are at a particularly high risk of cancer. A majority of the cases diagnosed on hormonal evaluation are ascribed ER, PR negative or triple-negative status, thus complicating treatment of the disease.

Early-onset breast cancer tends to have a more aggressive progression than late-onset cancer and hence, timely diagnosis is critical. Another clinically relevant difference is that while in the US, nearly $60-70 \%$ of all breast cancer patients are in stage-1, the figure hovers around $10 \%$ for Indians. On the other hand, the figures for patients presenting with stage-IV disease are $10 \%$ in the US, but for Indians, the statistics reveal the numbers to be around $25 \%$ and a further $50 \%$ with stage-III disease (Bhattacharyya et al., 2020). This was also supported by Leong et al., (2010) who published an article assessing differences in breast cancer presentations across the world and concluded that in India majority of the patients present at the stage of local advancement of the disease or with metastatic spread to other sites.

Understanding this demographic variation is of paramount importance, as it has major implications on the need for early screening programmes to assess for lesions in the breast which might potentially turn out to be neoplastic growths. Early screening can potentially allow for early diagnosis of breast cancer and thus shift the curve in favour of stage- 1 and 2 breast disease from current stage-3 and 4, thus allowing for relatively more conservative options, and improving patient prognosis. It can theoretically allow for an improvement in the 5-year survival rate for women diagnosed with breast cancer. The insights gained can contribute towards the formulation of policies that are more geared towards the Indian populace.

There are multiple radiological approaches to assess for a lesion in the breast, these include modalities like mammography, ultrasonography (USG) or MRI (Fiorica et al., 2016). Amongst them, MRI being an expensive modality cannot be used for screening purposes and is better utilised as an adjunct to support the diagnosis when suspicion arises on other modalities. While the USG is relatively inexpensive and is more easily available than a mammogram, it suffers from significant user subjectivity and a lack of personnel qualified enough to interpret it. The Somoinsight study, a multi-Institutional study undertaken between 2009 to 2011 involving 15318 females concluded that automated breast US is a useful tool best utilised as an adjunct to mammography in a generalisable cohort of women who have dense breasts, thus reiterating the usage of mammography for primary screening (Brem et al., 2015).

Hence, we performed screening at our tertiary care Institute using mammography and utilised digital breast tomosynthesis in cases where mammography revealed suspicious findings which could not be conclusively resolved on a mammogram. The 2-year long screening programme was augmented with three community outreach screening camps with the base at a tertiary care Institute in Western India. The programme was organised in the district of Pune which drains an estimated population of nearly 9 million (2021) (Indiacensus- city, Pune, Pimpri-chinchwad), which is greater than the individual population of over 50 world nations (Nationsonline- one world, country wise population). The district comprises of the wider Pune Metropolitan area and 13 other Talukas. Multiple outreach camps were organised in three different Talukas to ensure urban, rural and tribal population were reached adequately.

\section{Materials and Methods}

The screening programme was a prospective study performed over a period of nearly 2 -years between 
September 2019 to July 2021. The study was HIPAA compliant and approved by the Institutional Ethics Committee and Institutional Review Board of the tertiary care Institute. Full, free, voluntary, informed consent was sought from all the women involved in the programme.

\section{Criteria to include and exclude:}

All females above 40-years of age were included in the study (As per current established ACR guidelines, due to a lack of pre-existing India specific guidelines), those with additional risk factors predisposing them for breast cancer, were screened at an earlier age too.

We excluded known cases of breast cancer, patients sent with a clinically palpable breast lump, pregnant patients, males with breast disease.

\section{Institutional set-up for mammography}

In India, due to a lack of awareness about women's health and various social taboos, many women have a complete lack of knowledge of practices vital for good health. Most of them do not understand the risk factors which might predispose them to future breast cancer. Hence, there is, in general, a lesser than expected turn-out of women directly to the Hospital for screening purposes. With this concern in mind, the Institute appointed two awareness generation officers, whose sole purpose was to disseminate information and knowledge pertaining to the risk of breast cancer. These officers were themselves, middle-aged females, this was a necessity as the target populace would be more receptive to communication arising from other middle-aged women. These women workers were tasked with going to the waiting room of various Outpatient Departments (OPDs) with permanent posters and pamphlets and disseminate information to the target population. Once they had identified these women, they would in isolation ask them for any existing breast complaints, family history of breast and ovarian cancer, history of oral contraceptive use, age of attaining menarche and first child, history of breastfeeding, to get a fair idea about the associated risk factors.

\section{Camp based set-up for mammography}

If the entire population screened was one from the hospital, then there were chances that there would be associated bias and the screened population may not be representative of the actual population. Also, to inculcate actual behaviour change in the population, it is important to make them aware of "good practices". Towards this end, the Institute organises monthly camps in various Talukas under the Pune district. While these are general medical camps, knowledge pertaining to breast cancer and menstrual hygiene is provided to all those visiting these camps by means of posters, "nukkad natak", to sensitise them towards this issue. In addition to this, the Institute organised special camps focussed solely to generate interest in the screening programme. Two awareness generation officers were assigned to these camps, whose role was the same as in an Institution-based screening programme- disseminate information, but as an added role, they were to accompany willing females for the purpose of screening to the Institute. Those who gave consent for screening mammography were brought in a bus to the institute where screening was performed completely free of cost. In fact, those who were interested in receiving films and were willing to pay were charged a mere Rs110 (\$1.5), for 4 films.

These camps thus served the important role of providing the drainage population by creating awareness and a lack of charges provided the incentive to these women. A fixed-equipment model was used as the screening had to be performed using digital breast mammography, the equipment is expensive, costing significantly more than a conventional mammography machine. Three such camps were organised over a period of 2 years, in fact, the collective population screened through these camps accounted for a little less than 50\% of the entire screened population (Figure 1).

\section{Equipment used}

Mammography: The equipment used was a Siemens MAMMOMAT INSPIRATION with a plate size of $24 \times 30 \mathrm{~cm}$. The equipment is high-end and permits digital acquisition of mammograms along with tomosynthesis. Screening mammography entails a basic explanation to the patient of the procedure. The entire scan takes 5 minutes and if we include time to dress and undress, $10 \mathrm{mins}$. Once the patient is in a comfortable gown, the standard craniocaudal and mediolateral oblique projections are acquired.

\section{Digital Breast Tomosynthesis}

DBT Images were acquired on the same dedicated digital mammography unit with $\mathrm{x}$-ray tube rotation through an angular range of 15 degree ( -7.5 degree to +7.5 degree) with the breast in standard compression, in both MLO and CC projections, $25-49 \mathrm{kVp}$, max 200mA, selenium (Se) detector $24 \times 30 \mathrm{~cm}$, pixel size $140 \mathrm{um}$, PMMA Plate. Image acquisition was performed with a continuous exposure method (pulsed, short exposures during continuous motion of the X-ray tube), with an acquisition time of 5 seconds or less for one breast. Image reconstruction was performed immediately after image acquisition: Slice thickness $=1 \mathrm{~mm}$, time of reconstruction $=2-5$ seconds and a reconstructed pixel size of about 100um.

\section{Standardisation of technique}

To ensure that repeat mammograms were not needed, the technicians were trained to differentiate between an ideal and a less than an ideal mammogram. Towards this end, a strict point-based policy was employed for each view. This ensured that each view met the basic criteria and hence, the entire breast parenchyma was well assessed (Figure 2).

Craniocaudal view: It should allow visualisation of the majority of breast tissue over both medial and lateral aspects of the breast with also visualisation of the retro mammary space and some portion of pectoral muscles (Figure 2A).

Medio-Lateral-Oblique view: It should allow comprehensive evaluation of the axilla, axillary tail, and infra-mammary fold along with the breast parenchyma (Figure 2B). 


\section{Statistical Analysis}

The entire data was entered into a Microsoft Excel data sheet to form a master chart. The data was subsequently analyzed using SPSS 22 version software. Continuous data was represented as mean and standard deviation. Categorical data was represented in the form of frequencies and proportions. The Chi-square test was used as a test of significance for qualitative data.

Graphical representation of data- MS Excel and MS word were used to obtain various types of graphs such as bar diagrams, Pie diagrams.

P-value (Probability that the result is true) of $<0.05$ was considered as statistically significant after assuming all the rules of statistical tests.

Statistical software: MS Excel, SPSS version 22 (IBM SPSS Statistics, Somers NY, USA) was used to analyze data.

\section{Results}

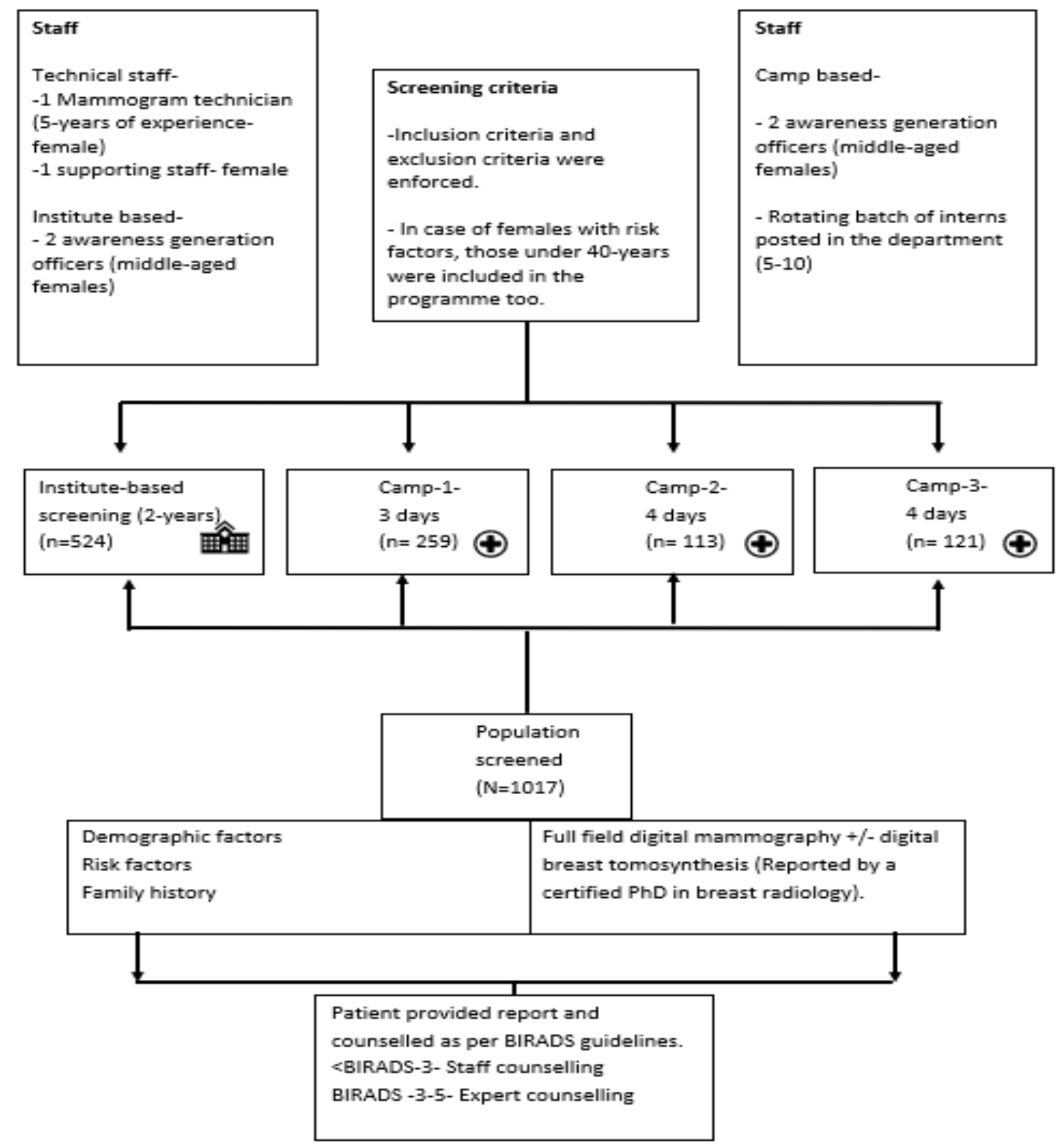

A demographic assessment of the age distribution of the women presenting for screening revealed the mean age of subjects to be $49.05 \pm 9.002$ years. Amongst them, the majority of the women (39\%) belonged to $41-50$ years of age group and another $20.5 \%$ of the women were 40 -years of age, $5.4 \%$ of the women were less than 40 -years of age and were found to have at least one high-risk factor and hence advised early screening. The next most common age group was 51-60years accounting for nearly a quarter (24\%) of all women. Elderly women (61-70 years) accounted was almost a tenth of all women; those over 70 years comprised only $1.2 \%$ of those screened.

Digital breast mammography revealed a significant number of women had dense breasts, nearly $43 \%$ had type-C breasts, while a further $29 \%$ had type- B breasts with scattered fibro-fatty tissue. Almost a quarter of the women had entirely fatty breasts (type-A). The remaining $4 \%$ had extremely dense breasts (Figure 3 ). It was also noted that up to 50 years of age, most women 


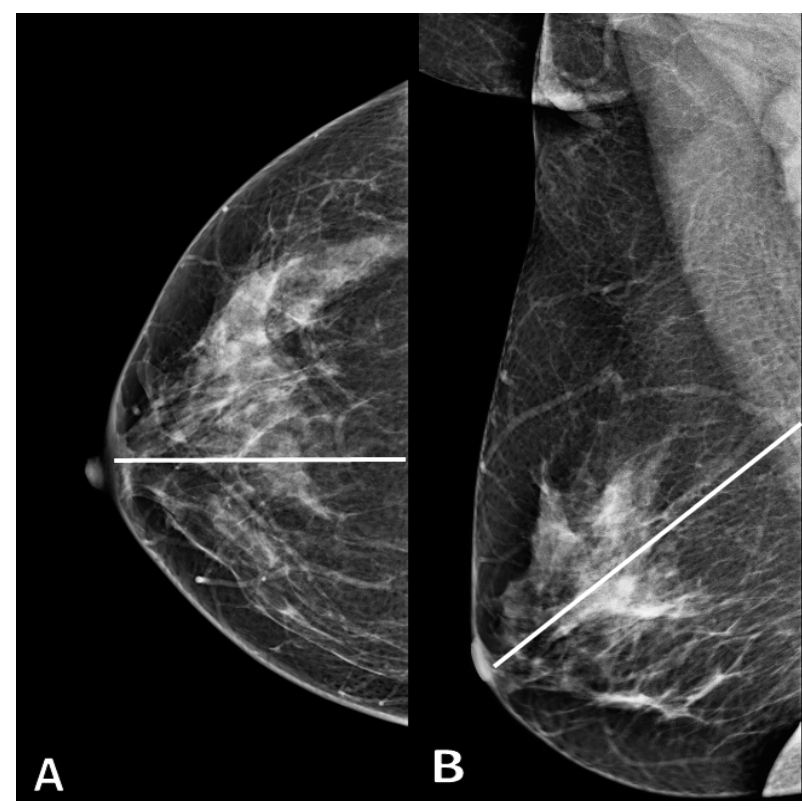

Figure 2. Mammography Reveals the Pectoralis-Nipple Line on A). CC view and B). MLO view.

(nearly $50 \%$ ) had type-C breasts. While between the ages 51-70years, type-B was the most common parenchymal density. Above, 70 years of age, most women had fatty (type-A) breasts.

Out of the 1,017 women screened, nearly $210(20.6 \%)$ of them had at least one lesion detected on at least one
Table 1. Side (Laterality) Distribution of the Lesion Detected on Mammogram

\begin{tabular}{llcc}
\hline & & Count & $\%$ \\
\hline Side & Left & 111 & $52.90 \%$ \\
& Right & 93 & $44.30 \%$ \\
& Bilateral & 6 & $2.90 \%$ \\
& Total & 210 & $100.00 \%$ \\
\hline
\end{tabular}

side. A breakdown of the distribution of the lesion is shown in Table 1. It reveals more lesions were detected on the left side than on the right. While only in $6(2.9 \%)$ of the women, were lesions detected on both sides. Nearly half of all these lesions (102) were less than $1.5 \mathrm{~cm}$ across in their longest dimension and around 70 were subcentimetric sized lesions. The distribution of the lesions in various age groups was nearly similar, ranging between $16.8 \%$ to $22.8 \%$, the difference of distribution across ages was not significant statistically.

Over half of all the lesions were detected in the upperouter quadrant $(52.4 \%)$, while around a quarter of the lesions were located in a retro mammary location (23.8\%). Another quarter of all the lesions were located in the inner quadrants of the breast (23.3\%). While the frequency of lesion detection in the lower outer quadrant was least at around $0.5 \%$ (Figure 4 ).

Overall, $3.5 \%$ of all the mammograms had microcalcification. Amongst women with a lesion detected

\section{Parenchymal Density distribution}

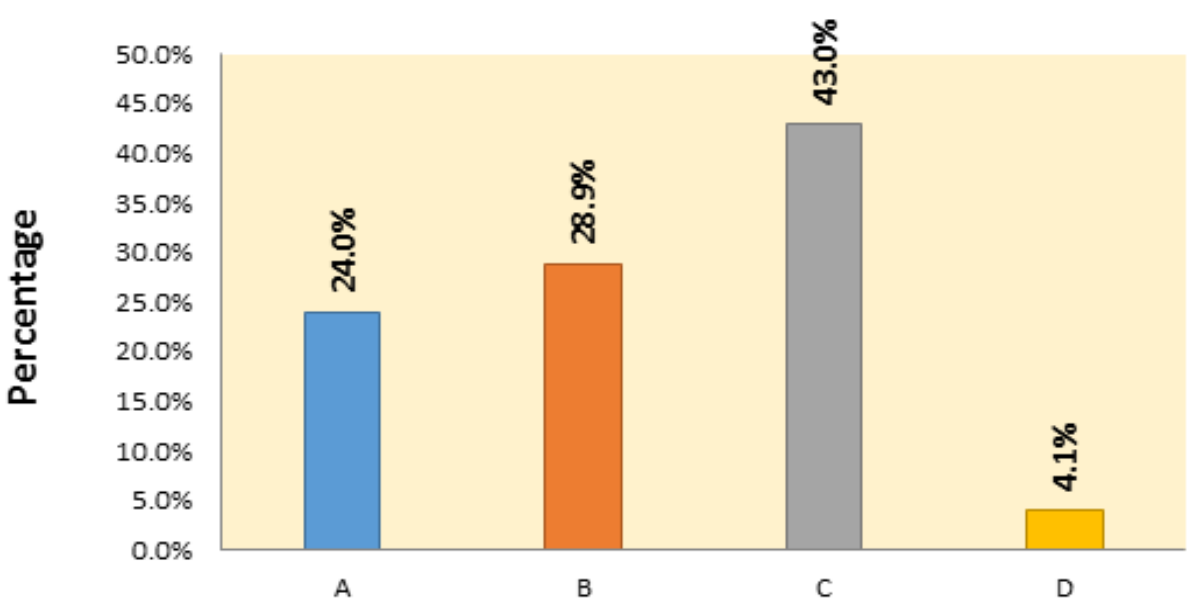

\section{Parenchymal Density (Type)}

Figure 3. Bar Diagram Showing Distribution of Parenchymal Density Across the Women Screened.

Table 2. Micro Calcification and Architectural Distortion among Subjects with Mass

\begin{tabular}{lcccccc}
\hline & & \multicolumn{2}{c}{ Mass (Lesion) } & \multicolumn{2}{c}{ Pbsent } \\
& & \multicolumn{2}{c}{ Present } & & \\
& & Count & $\%$ & Count & $\%$ & \\
\hline Micro calcification & Present & 29 & $13.80 \%$ & 7 & $0.90 \%$ & $<0.001^{*}$ \\
& Absent & 181 & $86.20 \%$ & 800 & $99.10 \%$ & \\
\multirow{3}{*}{ Architectural Distortion } & Present & 18 & $8.60 \%$ & 12 & $1.50 \%$ & $<0.001 *$ \\
& Absent & 192 & $91.40 \%$ & 795 & $98.50 \%$ & \\
\hline
\end{tabular}




\section{LOCATION OF LESION DISTRIBUTION}

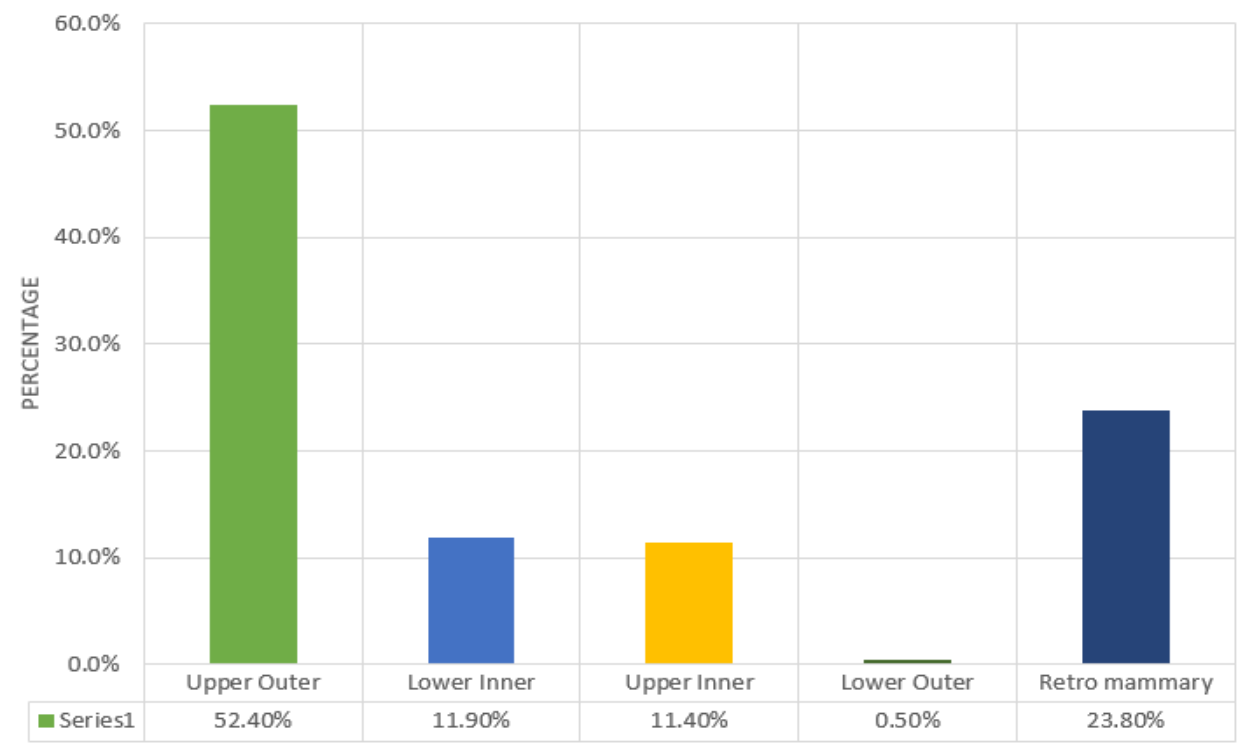

Figure 4. Bar Diagram Showing Quadrant-Wise Distribution of the Detected Lesions

on mammography, $13.8 \%$ of them had microcalcifications. Similarly, architectural distortion was detected on $2.9 \%$ of all mammograms. Amongst women with a lesion detected on mammography, $8.6 \%$ had associated architectural distortion (Table 2). Therefore, both microcalcification and architectural distortion were found to be significantly associated with the presence of a mass (lesion).

Focal asymmetry was seen in only $18(1.8 \%)$ women. Axillary lymphadenopathy was noted in 310 (30.5\%) women. Benign calcifications like vascular, rod-like, round with central lucency, macrocalcification were noted in 186 women (18.3\%) (Figure 5).

Our analysis of breast categories based on BI-RADS criteria (Figure 6) revealed that the majority of women (57\%) did not have any detectable abnormality and were disease-free (BIRADS-1). BIRADS-2 was the next most frequently assigned category and had mammographically detected pathologies that were conclusively benign (BIRADS-2), these included benign lesions and benign calcifications (Figure 7). A minority of lesions were assigned BIRADS-3 category, which is probably benign and were advised to follow-up. BIRADS-4 and 5,

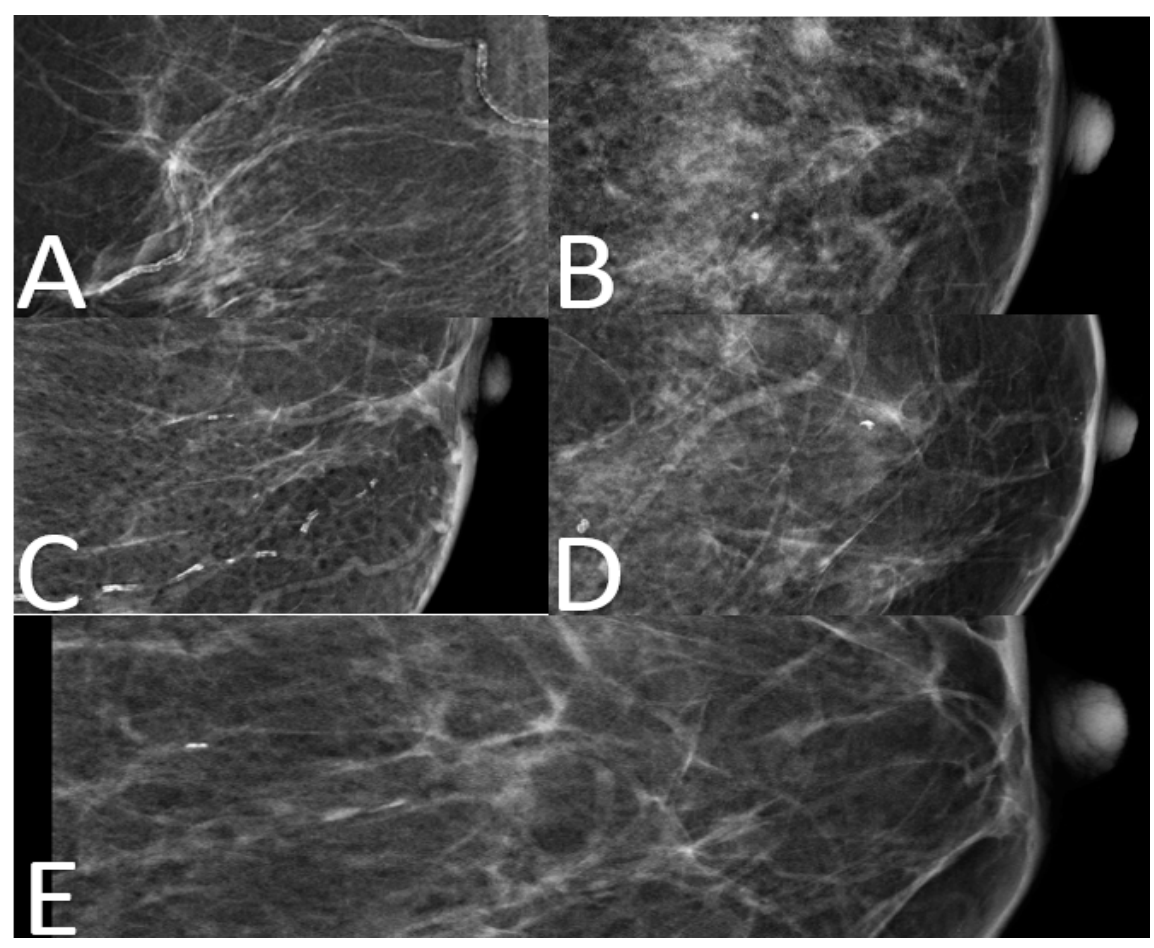

Figure 5. Few of the Patterns of benign Calcifications Seen on Digital Breast Mammography- Continuous Vascular Calcification (A); Macro-calcification seen within the breast parenchyma (B); Discontinuous "tram-track" vascular (C), Multiple round calcifications with lucent center and with skin calcification (D); Rod-like calcification (E). 


\section{Final BIRADS Score Distribution}

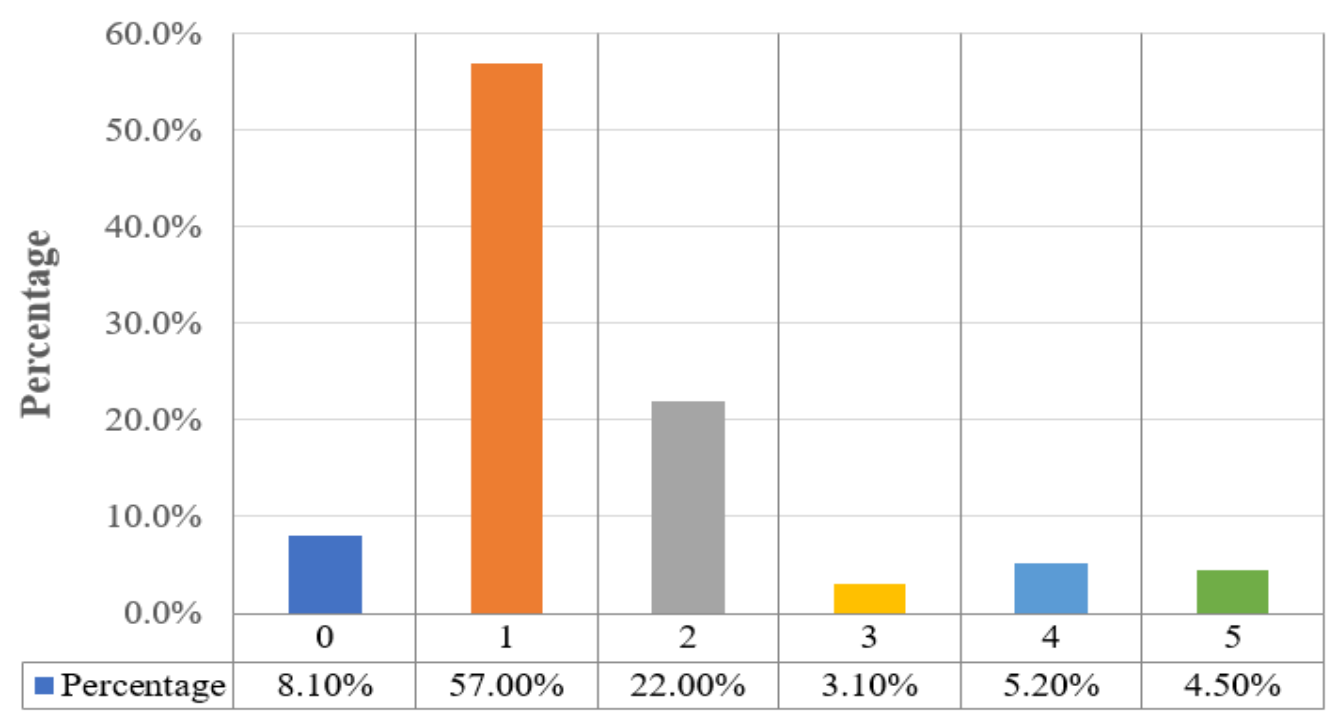

Figure 6. Bar Diagram Showing the Population Distribution as Per assigned BIRADS Categories.

collectively accounted for nearly $10 \%$ of all cases (Figure 8).

Out of all BIRADS-5 assigned lesions on mammography, 5 were lost to follow-up and 3 refused to undergo follow-on diagnostic investigations. Amongst the women who agreed to undergo biopsy, all except 2 were malignant on histopathological correlation. The other two turned out to be benign fibro-adenosis.

In the third screening camp organised, a questionnaire was provided to the participating women $(n=121)$ to assess certain risk factors predisposing them to breast cancer. The analysis revealed that the mean age at menarche in these women was 12.2 years, while the range was $11-18$ years with the mode being 12 . Most of the women had at least 2 children and therefore the overall period of breastfeeding was between 1-2 years in most of them. Six of the women had a first degree relative with breast cancer, out of which 4 had a history of breast cancer in their mother, while 2 had a history in both mother and sister. They did not know about the status of the BRCA gene and whether testing had been performed on them or not. None of them had any history of ovarian carcinoma.

\section{Discussion}

Breast cancer is a continually rising problem in developing countries like India, in part due to a change in lifestyle factors like dietary modification and age of childbearing. Another major hurdle is that in Developing countries like India, the lesions are often more advanced

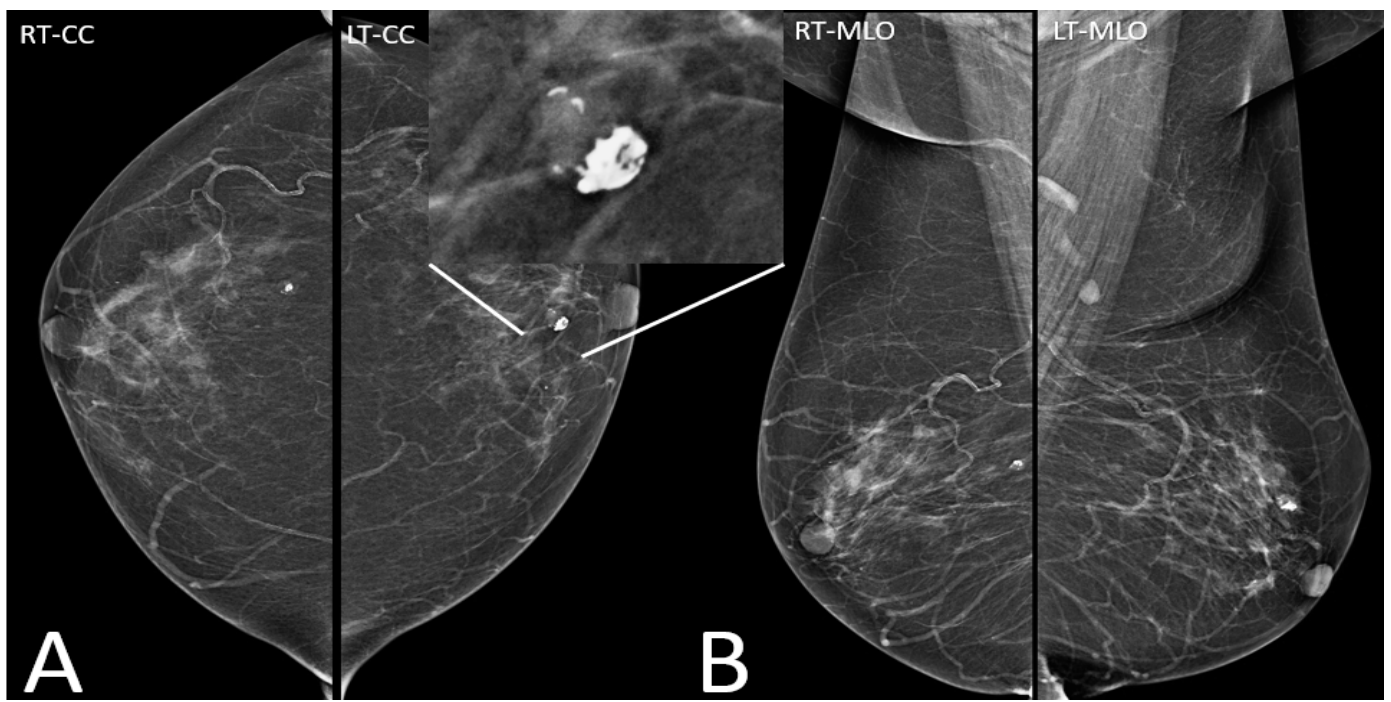

Figure 7. Screening Digital Mammogram of a 58-Year-Old Woman Revealed Presence of a Small Calcified Lesion in the Retromammary Region of Right Breast and another Similar Lesion with Popcorn Calcification (Zoomed-in view) is Noted in the Left upper-outer Quadrant (CC and MLO views). These were labelled as involuting fibroadenomas and consequently assigned BIRADS-2. 




Figure 8. Screening Digital Breast Mammogram of a 48-Year-Old Woman Reveals an Ill-Defined Density Mass in the Upper Outer Quadrant of Left Breast (A) CC and (B) MLO Views. Pleomorphic microcalcification seen over the mass lesion. The lesion was confirmed utilising digital breast tomosynthesis and assigned BIRADS-5. Histopathology confirmed the lesion as malignant.

when first detected, consequent to their delayed presentation (Kumar et al., 2017). The factors contributing to their delayed presentation are multi-pronged, ranging from an overall lack of social awareness particularly in tier-2 and 3 cities, towns, villages, which collectively account for a large majority of the population, thus there is a significant part of the population who do not recognise the potential implications of early breast mass detection (Wu et al., 2019; Salama, 2020). In India, socio-cultural factors are a big barrier for women; those who do present to healthcare centres are often stigmatised (Thakur et al., 2015). Women cannot discuss with appropriate healthcare professionals' problems pertaining to menstruation and breast complaints, as their families and community restrict them from doing so (Khokar et al., 2015).

Another bottleneck remains a lack of adequate healthcare infrastructure and a lack of certified healthcare individuals. Equipment for mammography is not easily accessible due to a lack of fixed consoles in district and community hospitals. However, as demonstrated by pilot projects undertaken by Sayed et al., (2016) in Kenya, Kumar et al., (2017) in India, organising breast screening camps may obviate the necessity of having fixed equipment and thus the need to maintain the associated facilities. As observed by Kumar et al., (2017) through their camp, the number of people who they reached out to and the number of women they screened during a week-long camp was comparable to the population who presented to their hospital over a span of 6 months for the same.

In our study of 1,017 persons screened, using digital breast mammography in a tertiary care institution, we found that the majority of the population had heterogeneously dense breasts (type-C) followed by those with scattered fibro-fatty glands (type-B) and completely fatty breast parenchyma (type-A). Only a small minority had extremely dense breasts (type-D).

Most of the patients who underwent screening belonged to the 41-50 year age group with another significant proportion belonging to 31 to 40 years of age (35-40 years). The higher proportion of data appearing in this category is because of a lack of literacy in the population, a lot of the women who fall between the age of 40 to 41 years label themselves as 40 for ease of remembrance of age and were hence ascribed to this category. Nearly, 209 cases in our entire screening population were aged 40 as per their verbal record. Only 44 cases were actually younger than 40 years of age, out of which nearly 7 were categorised as BIRADS- 4 and a further 4 cases as BIRADS-5 lesions. If only less than the 40years of age group was a category, then this would actually account for only $4.3 \%$ of all cases. These 44 cases had been advised early screening due to the presence of at least one high-risk factor detected during breast camps.

The prevalence of various BIRADS categories in our study was BIRADS-1 followed by BIRADS-2, trailed by BIRADS- 4 and 3 respectively. BIRADS- 5 was least common amongst all the categories and nearly $8 \%$ of all scans were classified at BIRADS- 0 , that is the assessment was incomplete and needed additional views and imaging to further evaluate class. In a study undertaken by Joshi et al., (2017) to evaluate 362 women, BIRADS- 2 was most common, marginally more than BIRADS -1 , followed by BIRADS-3,4 and 5.

Amongst the population screened, nearly 20.6\% (210 women) of the population had a mass lesion detected on mammography. Overall tomosynthesis was performed in nearly 84 cases where the findings were suspicious on a digital mammogram. The majority of these lesions were classified into BIRADS categories 4 and 5 . Over $70 \%$ of lesions classified as BIRADS- 4 and 5 and nearly half of lesions classified as BIRADS-3 had undergone digital 
breast tomosynthesis to conclusively assign them an ACRBIRADS category. We could not find any prior relevant published data in this regard.

Lesions in our study were most frequent in the upper outer quadrant, followed by the retromammary region; the upper inner and lower inner quadrants were the next most frequently involved regions, occurring with nearly the same frequency; while the lower outer quadrant was least frequent. Our findings are consistent with previous research by Chan et al., (2017) and Yadav et al., (2018) in their cohort of 110 women who found that the upper outer quadrant was the most common location of the lesions.

Architectural distortion is the third most common sign on mammography of breast cancer which is clinically not palpable (Rangayyan et al., 2010). While the main goal with assessing architectural distortion is to utilise it as an indirect sign of malignancy, nonetheless it is also seen in certain benign conditions like radial scars and complex sclerosing lesions (Durand et al., 2016). In our case, distortion was seen in nearly $2.9 \%$ of the entire population and an increase in their frequency of appearance was associated with a higher BIRADS category, and this increment was statistically significant. In our study population, there were 10 cases where the woman did not have any mass detected on mammography but had architectural distortion conclusively detected on a full-field digital mammogram. In only three cases was architectural distortion characterised solely on digital tomosynthesis.

Focal asymmetry of breast lesions can be ascribed to a variety of benign or malignant causes; these range from fibrocystic disease, breast necrosis, fibroadenoma, tubular ectasia on the benign spectrum to invasive carcinoma, ductal carcinoma in-situ and primary lymphoma on the malignant spectrum (Chesebro et al., 2016). In our screening population, focal asymmetry was observed in nearly $1.8 \%$ of all women. Our figures are slightly lower than that reported by Sickles (1998) in their large study assessing 61,273 two-view mammograms published in 1998 . They reported the presence of asymmetry in nearly $3.3 \%$ of all screening mammograms. The difference may be ascribed to our usage of digital mammography along with digital breast tomosynthesis to characterise the breast parenchyma, as research has proven that while overall digital mammography is comparable to a conventional screening mammogram, but it has primacy over conventional detection in dense breasts. This has been established in a large cohort study performed by Kerlikowske et al., (2011) involving 329,261 women. Similar conclusions were also drawn in a review performed by Faridah (2008). Out of the 18 women with focal asymmetry, 8 of them had dense breasts (type-C).

Amongst the population with mass, the majority of them had lesions on a single side with the percentage being slightly higher on the right side than the left. However, this difference was not significant statistically. However, nearly $3 \%$ of the cases had lesions in both breasts which accounted for $0.5 \%$ of the screened population. Our findings were in accord with other previously published studies for breast cancer; Amer et al., (2014) in their study encompassing 687 breast cancer patients revealed that overall left-sided breast cancer was more common than the right side by nearly $5 \%$ while bilateral cancers were seen in nearly $2.9 \%$ of cases, our figures exactly match this. Tulinius et al. (1990) in their study encompassing 2,139 cases, concluded that breast cancer was more common on the left than the right by nearly $13 \%$.

Microcalcification was detected in $3.5 \%$ overall when considering the entire population, but in $13.5 \%$ (29 cases) of all lesions while in 7 cases $(0.9 \%$ of the entire population), they were found even without the presence of a mass lesion. They were seen to occur with increasing frequency in the higher BIRADS category and this increment was statistically significant. Microcalcifications are an independent risk factor for breast cancer and therefore their presence in a screening mammogram should raise a higher degree of suspicion and a further detailed evaluation (Azam et al., 2021). They are of particular significance in cases where there are no clinically palpable lesions.

The primacy of a well thought out, and enforced screening campaign for breast cancer, tailor-made keeping in sight the countries population and constraints would be to have long term improvement in survival rates as more patients will be diagnosed early, thus the advantageous down-staging of the disease. This is supported by the research performed by Chuwa et al., (2009) through the first-ever population-based breast screening programme in Singapore (BSS). They concluded that screen-detected lesions were particularly smaller when compared to patients presenting with symptomatic lesions. Also, there was the dual advantage that screening by allowing early detection allowed treatment when the disease was in a relatively early stage and thus consequently permits relatively more conservative approaches. A similar claim was supported by Agrawal et al., (2014) in their review of screening for breast cancer. They concluded that detecting lesions when they are small and have not spread to nearby lymph nodes will have a strong treatment impact and hence, screening has more benefits than risks and should be employed in the general population. The major challenge lies in cost assessment and bearing for such a mammoth programme, this was voiced by Reddy et al., (2012) in their assessment of the feasibility of mammograms in the Indian population. They concluded that due to a lack of trained staff the current cost of organising camps is prohibitive to including mammograms for primary screening and instead clinical breast examination should be preferred for phase- 1 and once capacity building and awareness has been generated can the second phase of mammography be included. However, Kumar et al., (2017) argued against such an approach stating that the benefits of including mammography from the start itself outweigh the associated costs and a sensitive Government should understand this aspect.

The debate for the usage of mammography alone versus clinical breast examination alone, particularly in lower-middle-income countries has been a particularly protracted one still unclear evidence. Early advocates of the screening programme like Baines and Miller (1997) concluded that while stand-alone mammography tends to be better at detecting neoplastic lesions, the actual benefit 
lies in clubbing both of them particularly for women in the age group between 40-49 years of age. Oestreicher et al. (2005) in their study published in 2005, however, advised caution in this regard as while clinical breast examination has an incremental role to play in cancer detection to mammography, it also increases the overall number of false positives. In a recently concluded cluster randomised clinical trial published in BMJ by Mitra et al., (2021), encompassing a sample size of 1,50,000 women, distributed between test and control groups. The researchers established that breast examination reduced mortality by early diagnosis and the findings are statistically significant for women over 50 years of age, thus, establishing the basis for breast examination as a reliable alternative to mammography without much overdiagnosis. However, whether the incorporation as a sole method of screening at a population level translates into actual decrement of patient mortality has not been established and it will take further many years of surveillance to establish. In an overview analysing 11 systematic reviews performed between the years 1993 to 2019 by Ngan et al., (2020), the researchers concluded that despite clinical breast examination having a lower sensitivity than mammography, it achieved the same effect as mammography in terms of mortality. While this evidence is derived from indirect evidence and till date there exists no direct evidence, it is significant in terms of the ease with which it can be performed and the wider availability of such an approach. Thus, deeming it favourable in lower-middle-income countries. Meanwhile, the American Cancer Society in its recommendations as recently as April 2021 recommends either mammogram or digital breast tomosynthesis for screening citing a yet unclear benefit from breast examination- clinical or self (Cancer org- breast cancer, screening tests and early detection of breast cancer). In women whose tumours were diagnosed early by mammography, they had $22 \%$ higher five-year survival rate vis-a-vis women who did not undergo mammography (Acha et al., 2009).

Our study had a relative limitation that it is predominantly representative of women from Western India and to truly assess the burden of breast cancer in India, more such studies in various parts of India are needed, we partly tried to offset the bias involved in screening solely opportunistic women presenting to hospitals, by organising camps across different municipalities in geographically distributed regions. The patients were incentivised by offering free mammogram reports and hence, the population turnout was high. While critics may argue that offering mammograms free of cost may not be sustainable in the long term especially when the associated infrastructure (machine) is expensive. Our study establishes that in fact on the contrary screening mammography can be a highly subsidised, self-sustainable programme, as the patients who are ascribed higher category on BIRADS based on digital mammography, need to undergo further investigation and treatment. Hence, while the community benefits from early detection and treatment of breast cancer the programme pays for itself by allowing the hospitals to sustain by charges associated with detailed investigations and treatment. This approach is particularly viable in tier- 1 and 2 cities that have the infrastructure to treat such patients, their reach can be enhanced by means of breast cancer awareness camps. For more remote, smaller towns, villages though an alternative strategy may be needed. While we performed our study using equipment enabled with digital mammography and tomosynthesis as we had the infrastructure available and digital mammography offers a better lesion detection rate than conventional setups, in more resource-constrained institutions, conventional mammography may be a reasonable, acceptable alternative.

\section{Author Contribution Statement}

Pranav Ajmera: Conceptualisation, Methodology, Data curation, Writing- draft preparation, Reviewing, Editing, Formal analysis, Investigation, Visualisation. Project administration. Pratiksha Yadav: Conceptualisation, Methodology, Data curation, Writing- draft preparation, Reviewing, Editing, Formal analysis, Investigation, Visualisation. Project administration. Udayan Dosi: Data curation, project administration. Shreeya Goyal: Data curation.

\section{Acknowledgements}

We would like to acknowledge the support provided by Dr. Bhagyashree Patil (Pro-chancellor), Dr Yashraj Patil (Treasurer), Dr J.S. Bhawalkar (Dean), Dr.Amarjit Singh (CEO) of our institute, for making available the hospital resources, including vehicle and equipment, creating additional staff positions for better coordination of this large-scale project and organisation of camps. We would like also to acknowledge the departments of surgery, oncosurgery and pathology of the institute, for their help with follow-up of the patients and evaluation of diagnosis. The efforts of the residents and interns posted at the camps too deserve recognition.

\section{Approval Statement}

The project was approved by the institutional ethics committee of Dr DY Patil Medical College, Hospital and Research Center, Pune, as part of student thesis of the primary author (Pranav Ajmera). The approval number for the research project is IESC/PGS/2019/164 dated September, 2019.

\section{Ethical Issue}

The research project was approved by the institutional ethics committee of Dr DY Patil Medical College, Hospital and Research Center, Pune. The approval number for the research project is IESC/PGS/2019/164 dated September, 2019.

\section{Data Availability}

The individual patient data is archived in our hospitals server. While the data is accessible to the institute, for reasons of patient confidentiality the entire data and patient specific details cannot be made public.

\section{Conflict of Interest}


None of the authors have any conflict of interest to declare.

\section{References}

Acha B, Serrano C, Rangayyan R, Desautels JE (2009). Detection of microcalcifications in mammograms. $J$ Electronic Imaging, 18, 013011

Agarwal G, Pradeep PV, Aggarwal V, et al (2007). Spectrum of breast cancer in Asian women. World J Surg, 31, 1031-40.

Agrawal A, Tripathi P, Sahu A, Daftary J (2014). Breast screening revisited. J family Med Primary Care, 3, 340.

Amer MH (2014). Genetic factors and breast cancer laterality. Cancer Manag Res, 6, 191.

Azam S, Eriksson M, Sjölander A, et al (2021). Mammographic microcalcifications and risk of breast cancer. $\mathrm{Br} J$ Cancer, 14, 1-7.

Baines CJ, Miller AB (1997). Mammography versus clinical examination of the breasts. Cancer Inst Monogr, 22, 125-9.

Bhattacharyya GS, Doval DC, Desai CJ, et al (2020). Overview of breast cancer and implications of overtreatment of early-stage breast cancer: An Indian Perspective. JCO Glob Oncol, 6, 789-98.

Brem RF, Tabár L, Duffy SW, et al (2015) Assessing improvement in detection of breast cancer with three-dimensional automated breast US in women with dense breast tissue: the SomoInsight Study. Radiology, 274, 663-73.

Cancernet- cancer types, breast cancer, statistics, n.d. (WWW Document) (URL: https://www.cancer.net/cancer-types/ breast-cancer/statistics ) (Accessed: 9.10.2021).

Chan S, Chen JH, Li S, Chang R, et al (2017). Evaluation of the association between quantitative mammographic density and breast cancer occurred in different quadrants. $B M C$ cancer, 17, 1-1.

Chesebro AL, Winkler NS, Birdwell RL, Giess CS (2016). Developing asymmetries at mammography: a multimodality approach to assessment and management. Radiographics, 36, 322-34

Chopra B, Kaur V, Singh K, et al (2014). Age shift: Breast cancer is occurring in younger age groups-Is it true?. Clin Cancer Investig, 3, 526.

Chuwa EW, Yeo AW, Koong HN, et al (2009). Early detection of breast cancer through population-based mammographic screening in Asian Women: A Comparison Study between Screen-Detected and Symptomatic Breast Cancers. Breast $J, 15,133-9$.

Dhillon PK, Mathur P, Nandakumar A, et al (2018). The burden of cancers and their variations across the states of India: the Global Burden of Disease Study 1990-2016. Lancet Oncol, 19, 289-306

Durand MA, Wang S, Hooley RJ, Raghu M, Philpotts LE (2016). Tomosynthesis-detected architectural distortion: management algorithm with radiologic-pathologic correlation. Radiographics, 36, 311-21.

Faridah Y (2008). Digital versus screen film mammography: a clinical comparison. Biomed Imaging Interv $J, 4, \mathrm{e} 31$

Fiorica JV (2016). Breast cancer screening, mammography, and other modalities. Clin Obstet Gynecol, , 59, 688-709.

Joshi P, Sharma R (2017) Benign Lesions on Screening Mammography: Increasing Diagnostic Confidence in a Hitherto Unscreened Population. J Clinical Diagnostic Res, 11, TC14.

Indiacensus- city, Pune, Pimpri-chinchwad (WWW Document) (URL: https://www.indiacensus.net/city/pimpri-andchinchwad) (Accessed: 9.16.2021).

Kakarala M, Rozek L, Cote M, Liyanage S, Brenner DE (2010). Breast cancer histology and receptor status characterization in Asian Indian and Pakistani women in the US-a SEER analysis. BMC Cancer, 10, 1-8.

Kerlikowske K, Hubbard RA, Miglioretti DL, et al (2011). Comparative effectiveness of digital versus film-screen mammography in community practice in the United States: a cohort study. Ann Intern Med, 155, 493-502.

Khokhar A(2015). Study on knowledge, experiences and barriers to mammography among working women from Delhi. Indian J Cancer, 52, 531.

Kumar JU, Sreekanth V, Reddy HR, et al (2017) Screening mammography: a pilot study on its pertinence in Indian population by means of a camp. J Clinical Diagnostic Res, 11, TC29.

Leong SP, Shen ZZ, Liu TJ, et al (2010). Is breast cancer the same disease in Asian and Western countries?. World J Surg, 34, 2308-24.

Mathur P, Sathishkumar K, Chaturvedi M, ICMR-NCDIR-NCRP Investigator Group. (2020). Cancer statistics, 2020: report from national cancer registry programme, India. JCO Glob Oncol, 6, 1063-75.

McDonald JA, Rao R, Gibbons M, et al (2021). Symposium report: breast cancer in India- trends, environmental exposures and clinical implications. Cancer Causes Control, 28, 1-9.

Mittra I, Mishra GA, Dikshit RP, et al (2021). Effect of screening by clinical breast examination on breast cancer incidence and mortality after 20 years: prospective, cluster randomised controlled trial in Mumbai. BMJ, 24, 372.

Nationsonline- one world, country wise population (WWW Document). URL: https://www.nationsonline.org/oneworld/ population-by-country.html (Accessed: 9.16.2021).

Ngan TT, Nguyen NT, Van Minh H, Donnelly M, O’Neill C (2020). Effectiveness of clinical breast examination as a 'stand-alone' screening modality: an overview of systematic reviews. BMC Cancer, 20, 1-0.

Oestreicher N, Lehman CD, Seger DJ, Buist DS, White E (2005). The incremental contribution of clinical breast examination to invasive cancer detection in a mammography screening program. Am J Roentgenol, 184, 428-32.

Rangayyan RM, Banik S, Desautels JL (2010) Computer-aided detection of architectural distortion in prior mammograms of interval cancer. $J$ Digit Imaging, 23, 611-31.

Reddy N, Ninan T, Tabar L, Bevers T (2012). The results of a breast cancer screening camp at a district level in rural India. Asian Pac J Cancer Prev, 13, 6067-72.

Salama BM (2020). Factors affecting mammography screening utilization among educated women in Al Beheira governorate, Egypt. Indian J Community Med, 45, 522.

Sayed S, Moloo Z, Ngugi A, et al (2016). Breast camps for awareness and early diagnosis of breast cancer in countries with limited resources: A multidisciplinary model from Kenya. Oncologist, 21, 1138.

Sickles EA (1998). Findings at mammographic screening on only one standard projection: outcomes analysis. Radiology, 208, 471-5.

Sung H, Ferlay J, Siegel RL, et al (2021). Global cancer statistics 2020: GLOBOCAN estimates of incidence and mortality worldwide for 36 cancers in 185 countries. CA Cancer $J$ Clin, 71, 209-49.

Thakur NA, Humne AY, Godale LB (2015). Delay in presentation to the hospital and factors affecting it in breast cancer patients attending tertiary care center in Central India. Indian journal of cancer, 52, 102.

Tulinius H, Sigvaldason H, Olafsdottir G (1990). Left and right sided breast cancer. Pathol Res Prac, 186, 92-4.

WHO - News-room, factsheets, detail, breast cancer (WWW Document). (URL: https://www.who.int/news-room/fact- 
Pranav Ajmera et al

sheets/detail/breast-cancer) (Accessed: 9.10.2021).

Worldometers-info, world population, India population (WWW Document). (URL: https://bit.ly/3Ee7vL9) (Accessed: 9.15.2021).

Worldbank- indicator, Indian population (WWW Document) (URL: https://data.worldbank.org/indicator/SP.POP. TOTL?locations=IN) (Accessed: 9.15.2021).

Wu Z, Liu Y, Li X, et al (2019). Factors associated with breast cancer screening participation among women in mainland China: a systematic review. BMJ Open, 9, e028705.

Yadav P, Chauhan S (2018). Effectivity of combined diffusionweighted imaging and contrast- enhanced MRI in malignant and benign breast lesions. Polish J Radiol, 83, e82.

\section{c) (i) (8)}

This work is licensed under a Creative Commons AttributionNon Commercial 4.0 International License. 